\title{
Splenogonadal fusion evaluation using Contrast Enhanced Ultrasound and Elastography. A case report.
}

\author{
Sergio Grosu ${ }^{1}$, Johannes Rübenthaler ${ }^{1}$, Thomas Knösel², Matthias Trottmann³, \\ Julian Marcon ${ }^{3}$, Dirk-Andre Clevert ${ }^{1}$
}

${ }^{1}$ Department of Radiology, Interdisciplinary Ultrasound-Center, ${ }^{2}$ Department of Pathology, ${ }^{3}$ Department of Urology, University Hospital, LMU Munich, Munich, Germany

\begin{abstract}
We present the case of splenogonadal fusion in a 53-year-old male patient assessed by ultrasound and MRI, confirmed by pathologic examination. In addition to B-mode and colour-coded Doppler ultrasound, shear wave elastography and CEUS were performed and are presented in detail. Splenogonadal fusion is a rare congenital anomaly presumably caused by an abnormal attachment of splenic tissue to the gonad during gestation. Diagnosis is challenging for clinicians and in unclear cases splenogonadal fusion might cause unnecessary orchiectomies with benign pathologic results. Ultrasound is the first-line imaging modality in the diagnosis of testicular pathologies. This case report summarizes all available modern ultrasound imagingtechnologies and highlights the possibilities for the diagnosis of splenogonadal fusion.
\end{abstract}

Keywords: ultrasound; contrast-enhanced ultrasound; shear wave elastography; splenogonadal fusion

\section{Introduction}

Splenogonadal fusion is a rare congenital anomaly presumably caused by an abnormal attachment of splenic tissue to the gonad during the 5th to 8th week of gestation, resulting from the anatomical proximity of the spleen and the left gonad during stomach rotation. Consequently, in most cases it can be found on the left side of the testis and is an important differential diagnosis to other scrotal masses, especially testicular cancer. In unclear cases splenogonadal fusion might cause un-

Received 21.01.2019 Accepted 09.03.2019

Med Ultrason

2019, Vol. 21, No 3, 356-358

Corresponding author: Dr. med. Sergio Grosu, MD

Department of Radiology, University Hospital,

LMU Munich, Marchioninistraße 15,

81377 Munich, Germany

Phone: $+49894400-73620$

Fax: +49 89 4400-78832

E-mail: Sergio.Grosu@med.uni-muenchen.de necessary orchiectomies with benign pathologic results $[1,2]$.

Ultrasound (US) is the first-line imaging modality in the diagnosis of testicular pathologies. Technical advances such as elastography and contrast-enhanced ultrasound (CEUS) could add further value to this readily available imaging technique. Ultrasound elastography is assessing tissue stiffness. Having in mind that tumours mostly have a higher stromal tissue density, a reduced elasticity in comparison to surrounding tissue is an indicator for a malignant process [3]. CEUS provides complementary information to conventional ultrasound using intravenously administered ultrasound contrast agents. The time course of contrast enhancement of lesions can be compared to adjacent tissue allowing for an enhanced and continuous US evaluation of suspicious lesions. Therefore, CEUS has become a valuable instrument in the characterisation of scrotal masses [4].

This case report summarizes all available modern US imaging-technologies and highlights the possibilities for the diagnosis of splenogonadal fusion. 


\section{Case report}

A53-year-old male patient presented with unclear swelling of the left testicle. The patient had a history of orchiectomy of the right testicle after phlegmonous infection and abscess of the scrotum. The blood values showed elevation of the leucocyte level $\left(15200 / \mathrm{mm}^{3}\right)$ and C-reactive protein $(5.0 \mathrm{mg} / \mathrm{dl})$. Urine sediment was unremarkable. Screening parameters for testicular cancer were inconspicuous.

Initial native B-mode and colour-coded Doppler ultrasound showed a spherical hypoechogenic, hypervascular mass of $2.0 \times 2.1 \times 2.3 \mathrm{~cm}$ of the upper pole of the left testis. Additional shear wave elastography and CEUS of the left testis was performed using a Siemens S 2000 system (Siemens Healthineers, Erlangen, Germany) with linear array $9 \mathrm{MHz}$ multi-frequency transducers. The suspicious mass showed higher stiffness compared to the adjacent tissue. After a single injection of $2.4 \mathrm{ml}$ of SonoVue ultrasound contrast media followed by a flush of $10 \mathrm{ml}$ of $0.9 \% \mathrm{NaCl}$ solution, CEUS revealed a strong early-arterial hyper-enhancement of the suspicious lesion without wash-out in the late phase. In consideration of the high stiffness and strong early-arterial hyper-enhancement of the suspicious testicular lesion a splenogonadal fusion was suspected: however, a malignant lesion could not be ruled out (fig 1).

Additional MRI evidenced a suspicious mass of the upper pole of the left testis with homogenous contrast media uptake while the phlegmonous infection of the scrotum was significantly regredient (fig 2a). A malignant lesion could still not be completely ruled out.

Consequently, surgical scrotal exploration was recommended; the lesion was removed and the rest of the testis preserved. The pathologic examination of the specimen showed testicular parenchyma with a circumscribed, well-encapsulated lesion composed of predominantly red pulp and slightly reduced white pulp of splenic tissue, compatible with a discontinuous splenogonadal fusion. The specimen showed no sign of malignancy (fig $2 b$ ).

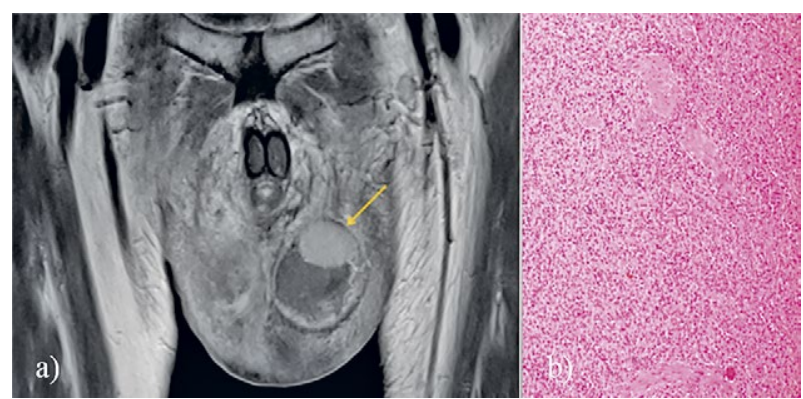

Fig 2. T1w MRI of the splenogonadal fusion (a) shows a wellcircumscribed mass of $2.6 \mathrm{~cm}$ x $2.4 \mathrm{~cm}$ with homogenous contrast media uptake of the upper pole of the left testis. Histological workup (hematoxylin and eosin stain, 10x magnification) of the splenogonadal fusion (b) after surgical resection shows normal splenic tissue in the testicle.

\section{Discussions}

Since the first report of ectopic gonadal splenic tissue by E. Bostroem in 1883, approximately 150 cases have been described. Most of these cases were incidental findings in groin exploitations of young patients under 30 years of age with a suspicion of pathologies such as epididymitis, testicular torsion or testicular tumour $[5,6]$.

Splenogonadal fusion is predominant in male patients with a ratio of circa 16:1 and can be classified into a continuous and discontinuous form. In cases of continuous splenogonadal fusion a splenic or fibrous cord between the spleen and the gonads can be found [2,7]. An association with other congenital malformations such as cryptorchidism, limb defects and micrognathia and in fewer cases also cardiac defects, spina bifida or cleft palate, was assessed in approximately $50 \%$ of all cases of continuous splenogonadal fusion. In contrast there is no known correlation of the discontinuous form of splenogonadal fusion with other congenital comorbidities. Malignancy in splenogonadal fusion is exceedingly rare, yet associated cryptorchidism may increase the risk of testicular cancer $[1,8]$.

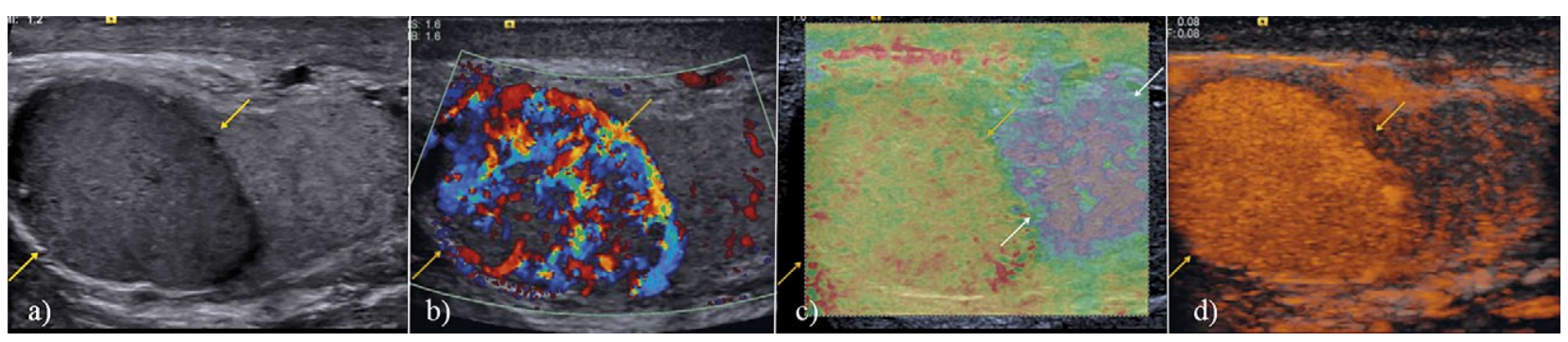

Fig 1. B-mode ultrasound (a) shows a spherical, hypoechogenic mass of the upper pole of the left testis (yellow arrows) with major vascularization in colour-coded Doppler ultrasound (b). Additional shear-wave ultrasound elastography (c) reveals a higher stiffness of the splenogonadal fusion (yellow arrows) compared to the adjacent tissue of the testis (white arrows). Contrast-enhanced ultrasound (d) shows a strong early-arterial hyper-enhancement of the splenogonadal fusion. 
US has an essential role in the diagnosis of scrotal masses as the first-line imaging modality in the diagnosis of testicular pathologies $[9,10]$. In B-mode splenogonadal fusion can be identified as a mass within the scrotal sac of low reflectivity, which may appear to be fused to the testis $[11,12]$. Colour-coded Doppler US shows a vascularisation pattern similar to that seen in the central aspect of the normal testis or in splenic tissue [13]. In elastography of splenogonadal fusion a higher stiffness compared to the adjacent tissue can be observed. CEUS reveals a characteristic arterial hypervascularization with contrast-pooling of the lesion in the late phase, similar to that of healthy splenic tissue [2].

In conclusion, we have described the diagnosis of splenogonadal fusion using shear wave elastography and CEUS of the testis in addition to B-mode and colourcoded Doppler ultrasound. CEUS and elastography are useful tools in helping to differentiate between testicular cancer and benign masses of the testis such as ectopic splenic tissue. Consequently, the value of US in the diagnosis of testicular pathologies can be increased even more by the additional use of these techniques.

\section{References}

1. Lopes RI, de Medeiros MT, Arap MA, Cocuzza M, Srougi M, Hallak J. Splenogonadal fusion and testicular cancer: case report and review of the literature. Einstein (Sao Paulo) $2012 ; 10: 92-95$.

2. Trottmann M, Marcon J, Mai V, et al. Characterization of splenogonadal fusion by contrast-enhanced ultrasound (CEUS) and elastography. Ultraschall Med. 2015;36:97-100.
3. Sigrist RMS, Liau J, Kaffas AE, Chammas MC, Willmann JK. Ultrasound Elastography: Review of Techniques and Clinical Applications. Theranostics 2017;7:1303-1329.

4. Schöppler G, Heinzelbecker J, Michaely HJ, Dinter D, Clevert DA, Pelzer AE. The impact of ultrasound in urology. Urologe A 2012;51:81-98.

5. Khairat AB, Ismail AM, Splenogonadal fusion: case presentation and literature review. J Pediatr Surg 2005;40:13571360.

6. Karray O, Oueslati A, Chakroun M, et al. Splenogonadal fusion - a rare cause of scrotal swelling: a case report. J Med Case Rep 2018;12:172.

7. Putschar WG, Manion WC. Splenicgonadal fusion. Am J Pathol 1956;32:15-33.

8. Lin CS, Lazarowicz JL, Allan RW, Maclennan GT. Splenogonadal fusion. J Urol 2010;184:332-333.

9. Cicero C, Bertolotto M, Hawthorn BR, et al. Multiple, Synchronous Lesions of Differing Histology Within the Same Testis: Ultrasonographic and Pathologic Correlations. Urology 2018;12:125-131.

10. Pozza C, Gianfrilli D, Fattorini G, et al. Diagnostic value of qualitative and strain ratio elastography in the differential diagnosis of non-palpable testicular lesions. Andrology 2016;4:1193-1203.

11. Henderson RG, Henderson DC, Reid IN, Atkinson PM. Case report: splenic-gonadal fusion--the ultrasound appearances. Clin Radiol 1991;44:117-118.

12. Kalomenopoulou M, Katsimba D, Arvaniti M, et al. Male splenic-gonadal fusion of the continuous type: sonographic findings. Eur Radiol 2002;12:374-377.

13. Stewart VR, Sellars ME, Somers S, Muir GH, Sidhu PS. Splenogonadal fusion: B-mode and color Doppler sonographic appearances. J Ultrasound Med 2004;23:10871090. 